\title{
artigo
}

Franco, C.M.M.A.; Bonfim, I.M.; Borges, R.M.; Lima, F.E.T.; Sousa, G.R.; Serra, K.S.

Uso de tecnologias educacionais baseadas na andragogia para educação de enfermeiros nefrologistas

\section{Uso de tecnologias educacionais baseadas na andragogia para educação de enfermeiros nefrologistas}

\author{
Use of educational technologies based on andragogy for education of nursing nurses \\ Uso de tecnologías educativas basadas en la andragogía para la educación de enfermeras de enfermería
}

\begin{abstract}
RESUMO
Objetivo: Analisar a produção científica acerca da utilização de tecnologias educacionais para treinamento e qualificação de enfermeiros nefrologistas que atuam na hemodiálise que tivessem como base a Andragogia. Método: 0 levantamento bibliográfico foi realizado por meio de consultas nas bases de dados: Literatura Latino-Americana e do Caribe em Ciências da Saúde (LILACS), SCIENCE DIRECT, MEDLINE, COCHRANE LIBRARY, BDENF e ERIC, com cruzamento de operadores. Resultados: Identificaram-se quatorze artigos, que revelaram uma variedade de tecnologias aplicadas ao ensino de Enfermeiros. Constatou-se que houve um predomínio dos estudos com força de evidência II e III, seguido no nível IV e V. Conclusão: Assim sendo, sugere-se na pesquisa a disseminação do conhecimento e construção de novos estudos sobre o tema a fim de melhorar a educação aos enfermeiros, seja na universidade, seja no seu ambiente de trabalho.

DESCRITORES: Enfermagem em Nefrologia; Tecnologia Educacional; Aprendizagem.
\end{abstract}

\section{ABSTRACT}

Objective: To analyze the scientific production on the use of educational technologies for training and qualification of nephrology nurses who work in hemodialysis based on Andragogy. Method: The bibliographic survey was carried out through consultations in the following databases: Latin American and Caribbean Literature on Health Sciences (LILACS), SCIENCE DIRECT, MEDLINE, COCHRANE LIBRARY, BDENF and ERIC, with crossing of operators. Results: Fourteen articles were identified, which revealed a variety of technologies applied to the teaching of nurses. It was found that there was a predominance of studies with strength of evidence II and III, followed by level IV and V. Conclusion: Therefore, it is suggested in the research the dissemination of knowledge and construction of new studies on the subject in order to improve education for nurses, whether at the university or in their work environment.

DESCRIPTORS: Nephrology Nursing; Educational Technology; Learning.

\section{RESUMEN}

Objetivo: Analizar la producción científica sobre el uso de tecnologías educativas para la formación y calificación de enfermeras nefrológicas que laboran en hemodiálisis basada en Andragogía. Metodo: El levantamiento bibliográfico se realizó mediante consultas en las siguientes bases de datos: Literatura Latinoamericana y del Caribe en Ciencias de la Salud (LILACS), SCIENCE DIRECT, MEDLINE, COCHRANE LIBRARY, BDENF y ERIC, con cruce de operadores. Resultados: Se identificaron catorce artículos que revelaron una variedad de tecnologías aplicadas a la enseñanza de enfermeras. Se encontró que hubo predominio de estudios con fuerza de evidencia II y III, seguidos del nivel IV y V. Conclusión: Por lo tanto, se sugiere en la investigación la difusión del conocimiento y la construcción de nuevos estudios sobre el tema con el fin de mejorar la educación de las enfermeras, ya sea en la universidad o en su entorno laboral.

DESCRIPTORES: Enfermería en Nefrología; Tecnología Educativa; Aprendizaje.

RECEBIDO EM: 28/06/2021 APROVADO EM: 06/07/2021

\section{Claudia Maria Marinho de Almeida Franco}

Mestre em Tecnologia e Inovação em Enfermagem pela Universidade de Fortaleza (UNIFOR)

ORCID: 0000-0003-1926-7592

\section{Isabela Melo Bonfim}

Mestre e Doutora em Enfermagem Clinico Cirúrgica pela Universidade Federal do Ceará- UFC. Docente em graduação em Enfermagem e do Mestrado Profissional Tecnologia e Inovação em Enfermagem da Universidade de Fortaleza - UNIFOR. CE-Brasil.

ORCID: 0000-0002-0056-862X 


\section{Rita Mônica Borges}

Doutora em Enfermagem pela Universidade Federal do Ceará. Especialista em Nefrologia e Bloco cirúrgico. Docente do Mestrado Profissional Tecnologia e Inovação em Enfermagem. CE-Brasil

ORCID: 0000-0002-5862-5244

\section{Francisca Elisângela Teixeira Lima}

Doutora em Enfermagem. Docente da Universidade Federal do Ceará-UFC.

ORCID: 0000-0002-7543-6947

\section{Gleison Resende Sousa}

Mestre em Tecnologia e Inovação em Enfermagem, Especialista em Urgência e Emergência. CE-Brasil. ORCID: 0000-0001-5805-9281

\section{Kalyni Silvino Serra}

Graduanda em enfermagem pela Universidade de Fortaleza - UNIFOR.

ORCID: 0000-0002-0542-4692

\section{INTRODUÇÃO}

A Andragogia, é a arte e ciência de conduzir adultos rumo ao aprendizado ${ }^{1}$, hgrupa princípios que contribuem para $\mathrm{o}$ sucesso da aprendizagem e, consequentemente, promovem melhoria na formação profissional, educação permanente e educação em saúde.

No final século XX, a Andragogia começou a ser utilizada na Enfermagem² ${ }^{2}$, razão pela qual é oportuno indagar sobre o atual estado de produção de conhecimentos nesse campo.

Segundo os dados a Sociedade Brasileira de Nefrologia (SBN) em 2018 no Brasil existe cerca de 786 centros de diálise com aproximadamente 119.850 pacientes em tratamento de hemodiálise ${ }^{3}$.

Dito isto, é de suma importância que para prestar uma assistência de enfermagem de qualidade o enfermeiro nefrologista deve manter sua qualificação através de aprendizado continuado,já quegrandes são os avanços tecnológicos naárea da nefrologia, principalmente na hemodiálise ${ }^{4}$.

Em tempos atuais onde as tecnologias educacionais estão cada dia inovando o processo de ensino aprendizagem, há uma necessidade dos enfermeiros nefrologistas apresentem eficácia nas unidades de diálise, para diminuir o tempo gasto nos treinamentos devido as muitas atividades elencadas a sua rotina de trabalho.

Nesse contexto surgiu o interesse pela temática, pois enquanto enfermeira nefrologista atuando em unidades de hemodiálise, observou-se que os enfermeiros tem grande dificuldade no desenvolvimento da educação continuada de sua equipe, com treinamentos cansativa e, por vezes demorados e ineficazes.

Destaca-se assim a relevância do estudo por permitir oferecer subsídios acerca das tecnologias educacionais utilizadas para o ensino de profissionais de enfermagem a fim de ajudar os profissionais de saúde, principalmente os enfermeiros, que atuam nessas unidades a conhecer um pouco mais sobre as oportunidades de educação e treinamentos com base no ensino para o adulto e as tecnologias educacionais atuais usadas para esse processo.

Nesse contexto, objetivou-se então analisar a produção científica acerca da utilização de tecnologias educacionais para treinamento e qualificação de enfermeiros nefrologistas que atuam na hemodiálise que tivessem como base a Andragogia, que é a ciência para o ensino do adulto.

\section{MÉTODO}

Trata-se de um estudo do tipo revisão integrativa, a qual tem a finalidade de reunir e sintetizar resultados de pesquisas sobre um determinado tema, de maneira sistemática e ordenada, contribuindo para o aprofundamento do conhecimento do tema investigado ${ }^{3}$.

Para o alcance do objetivo proposto, seguiram-se as seguintes etapas: seleção da amostra através da busca nas bases de dados; sumarização das informações extraídas dos artigos selecionados; avaliação dos estudos; interpretação e discussão dos resultados; apresentação da revisão e síntese do conhecimento 5 . O período de coleta foi entre março a julho de 2020, seguindo os critérios pré estabelecidos.
O levantamento bibliográfico foi realizado por meio de consultas nas bases de dados: Literatura Latino-Americana e do Caribe em Ciências da Saúde (LILACS), SCIENCE DIRECT, MEDLINE, COCHRANE LIBRARY, BDENF e ERIC. Utilizando os descritores: Nephrology Nursing AND Education, Higher AND Educational Technology de acordo com a terminologia MeSH, cruzando o operador booleano.

Os critérios de inclusão foram: artigos que contemplem os objetivos propostos, publicados nos últimos cinco anos, de outubro de 2014 a outubro de 2019, estar no idioma inglês, português ou espanhol; e estar disponível eletronicamente na íntegra.

Como critérios de exclusão foram adotados: a) estudos em formatos de editoriais; b) estudos em formato de cartas ao editor e c) revisões integrativas ou revisões de literatura.

Para a coleta inicial de dados utilizou-se um instrumento para busca de artigos já validados que analisa as características metodológicas dos estudos ${ }^{6}$. A revisão em pares ainda se mantém como o melhor método de revisão da pesquisa, podendo ser realizada de forma aberta, ou cega.

Dessa forma a equação de busca utilizada foi: Nephrology Nursing AND Education, Higher AND Educational Technology OR Education, Higher AND Educational Technology OREducational Technology AND Nephrology Nursing.

Sendo assim, foram identificados 6883 artigos sobre o tema proposto, sendo: $18 \mathrm{na}$ BDENF, 68 na LILACS, 12 na COCHRANE LIBRARY, 308 na MEDLINE, 160 na SCIENCE DIRECT e 6317 na ERIC. 


\section{artigo}

Franco, C.M.M.A.; Bonfim, I.M.; Borges, R.M.; Lima, F.E.T.; Sousa, G.R.; Serra, K.S.

Uso de tecnologias educacionais baseadas na andragogia para educação de enfermeiros nefrologistas

Após discussão dos achados, foram excluídos 6864 artigos, sendo: 18 na BDENF, 64 na LILACS, 12 na COCHRANE LIBRARY, 296 na MEDLINE, 160 na SCIENCE DIRECT e 6317 na ERIC por abordar outros temas, ficando assim fora dos critérios de inclusão. Foram selecionados: 04 na LILACS, 11 na MEDLINE, totalizando 15 artigos, após aplicação da elegibilidade, 01 estava duplicado, restante assim 14 artigos dentro dos critérios.

\section{RESULTADOS}

\section{DISCUSSÃO}

A leitura das obras selecionadas culminou em apreensões diversas acerca das estratégias utilizadas no processo de ensino aprendizagem em enfermagem através das tecnologias educacionais.

Constatou-se que houve um predomínio dos estudos com força de evidência II e III, seguido no nível IV e V, ou seja, pesquisas com níveis de evidências relacionadas a caso controle ou estudo de caso e originárias de estudo descritivo, respectivamente.

A1, A2, A5 e A14 autores enfatizam a importância da criação de novos panoramas educacionais para se buscar uma maior eficiência no processo ensino aprendizagem. Por outro, lado A4 autor diverge da afirmação e coloca que apenas a utilização de Team-Based Learning traz ao aluno o mesmo grau de obtenção de conteúdo.

Autores defendem o uso de tecnologias da informação e comunicação, bem como as tecnologias assistidas, para a melhoria no processo de ensino e aprendizagem ${ }^{20,21}$

Assis e Almeida ${ }^{20}$ relatam que a utilização das TICs, nas práticas pedagógicas são essenciais e de grande poder no que se refere ao

\begin{tabular}{|c|c|c|c|c|}
\hline ESTUDO & TÍTULO & $\begin{array}{l}\text { PAIIS DE ORIGEM/ } \\
\text { ANO DE PUBLICAÇÃO }\end{array}$ & MÉTODO & $\begin{array}{l}\text { NIIVEL DE } \\
\text { EVIDÊNCIA }\end{array}$ \\
\hline A1 & $\begin{array}{l}\text { A tendência do papel do professor no processo de aprendiza- } \\
\qquad \text { gem }^{7}\end{array}$ & 2018 / Brasil & $\begin{array}{l}\text { Pesquisa explora- } \\
\text { tória descritiva }\end{array}$ & 5 \\
\hline A2 & $\begin{array}{c}\text { Escala de estilos de aprendizagem em situações de uso de } \\
\text { tecnologias: estrutura interna }{ }^{8}\end{array}$ & 2018 / Brasil & $\begin{array}{l}\text { Estudo } \\
\text { Metdológico }\end{array}$ & 3 \\
\hline A3 & $\begin{array}{l}\text { Learning design e tecnologias: criação de ambientes colaborati- } \\
\text { vos para a aprendizagem }{ }^{9}\end{array}$ & 2017 / Brasil & Estudo Qualitativo & 4 \\
\hline A4 & $\begin{array}{l}\text { A Systematic Review Examining The Effectiveness Of Blending } \\
\text { Technology With Team-Based Learning }{ }^{10}\end{array}$ & 2016 / Austrália & $\begin{array}{l}\text { Revisão Sistemá- } \\
\text { tica }\end{array}$ & 2 \\
\hline A5 & $\begin{array}{l}\text { Competency And An Active Learning Program In Undergraduate } \\
\text { Nursing Education }{ }^{11}\end{array}$ & 2014 / USA & $\begin{array}{l}\text { Pesquisa Empírica } \\
\text { Quantitativa }\end{array}$ & 5 \\
\hline A6 & $\begin{array}{c}\text { Technology To Enhance In-Class Discussions And Student Parti- } \\
\text { cipation At A Multi-Campus Program }{ }^{12}\end{array}$ & 2019 / USA & $\begin{array}{l}\text { Pesquisa Transver- } \\
\text { sal Descritiva }\end{array}$ & 3 \\
\hline A7 & $\begin{array}{l}\text { Five Years Of Lesson Modification To Implement Non-Traditional } \\
\text { Learning Sessions In A Traditional-Delivery Curriculum: A Retros- } \\
\text { pective Assessment Using Applied Implementation Variables }\end{array}$ & 2017 / USA & COORTE & 3 \\
\hline A8 & $\begin{array}{l}\text { Flipping for success: evaluating the effectiveness of a novel } \\
\text { teaching approach in a graduate level setting }{ }^{14}\end{array}$ & 2015 / Canadá & Caso Controle & 4 \\
\hline A9 & $\begin{array}{c}\text { Improving nursing students' learning outcomes in fundamen- } \\
\text { tals of nursing course through combination of traditional and } \\
\text { e-learning methods }{ }^{15}\end{array}$ & 2019 / IRÃ & Caso Controle & 4 \\
\hline A10 & $\begin{array}{l}\text { The impact of assistive technology use for students with disabi- } \\
\text { lities in higher education: a systematic review }{ }^{16}\end{array}$ & 2019 / Irlanda & $\begin{array}{l}\text { Revisão Sistemá- } \\
\text { tica }\end{array}$ & 2 \\
\hline A11 & $\begin{array}{l}\text { An ehealth capabilities framework for graduates and health } \\
\text { professionals: mixed-methods study }{ }^{17}\end{array}$ & 2018 / Austrália & $\begin{array}{l}\text { Estudo Metodo- } \\
\text { lógico }\end{array}$ & 3 \\
\hline A12 & $\begin{array}{l}\text { Strategies used for the promotion of critical thinking in nursing } \\
\text { undergraduate education: a systematic review }{ }^{18}\end{array}$ & 2017 / USA & $\begin{array}{l}\text { Revisão Sistemáti- } \\
\text { ca da Literatura }\end{array}$ & 2 \\
\hline A13 & $\begin{array}{l}\text { Using bourdieu's theory of practice to understand ict use amon- } \\
\text { gst nurse educators }{ }^{19}\end{array}$ & 2014 / Reino Unido & $\begin{array}{l}\text { Estudo Explorató- } \\
\text { rio Descritivo }\end{array}$ & 5 \\
\hline A14 & $\begin{array}{l}\text { Docência em saúde: tempo de novas tecnologias da informação } \\
\qquad \text { e comunicação }{ }^{20}\end{array}$ & 2018 / Brasil & $\begin{array}{l}\text { Revisão Narrativa } \\
\text { de Literatura }\end{array}$ & 3 \\
\hline
\end{tabular}


processo de ensino, porém sem planejamento podemos ter a diminuição na adesão as mesmas, indicando a necessidade de desenvolver processos formativos com esse foco.

Segundo McNicholl ${ }^{21}$ através de revisão sistemática mostra a importância das AT (Tecnologia Assistidas) na formação acadêmica aumentando o aprendizado e o engajamento dos alunos, além de beneficiar a equipe de facilitadores acadêmicos.

Moraros ${ }^{14}$ descreve que a tecnologia Flipped Classroom teve como problema o manejo do programa e qualidade de áudio, o conforto dos alunos foi prejudicado devido ao atraso de postagem de vídeos.

Vemos através dos estudos mencionados na pesquisa que ainda temos um longo caminho para o desenho perfeito do processo de ensino aprendizagem para o profissional de enfermagem, como estudante em educação permanente.

\section{CONCLUSÃO}

Quando falamos em Enfermagem em Nefrologia, uma área tão específica de atuação, onde a tecnologia emerge com novos materiais e equipamentos em uma velocidade imensurável, vemos a necessidade urgente de melhorar a qualidade de ensino aprendizagem para aumentar a eficiência e diminuir o tempo de aprendizagem para a utilização dessas novas tecnologias.
A enfermagem em nefrologia, por ser uma área específica de atuação, onde a tecnologia emerge com novos materiais e equipamentos dia após dia em uma velocidade imensurável, vemos a necessidade urgente de melhorar a qualidade do processo de ensino aprendizagem para aumentar a eficiência e diminuir o tempo de aprendizagem para a utilização dessas novas tecnologias.

Faz-se então necessárias novas pesquisas sobre o tema para estimular os profissionais enfermeiros para a criação de novas tecnologias de ensino a fim de facilitar a educação continuada nas unidades de hemodiálise, melhorando assim a assistência aos pacientes renais.

\section{REFERÊNCIAS}

1. Knowles MS, Holton III EF, Swanson RA. Aprendizagem de resultados: Uma abordagem prática para aumentar a efetividade da educação corporativa. Rio de Janeiro. Elsevier; 2009.

2. Draganov PB, Friedlander MR, Sanna MC. Andragogia na Saúde: estudo bibliométrico. Esc. Anna Nery Rev. Enferm. 2011 jan-mar; 15 (1):149-156.

3. Sociedade Brasileira de Nefrologia. Censo SBN. Brasil: 2018. Disponível em: https:/sbn.org.br/categoria/censo-2018/. Acesso em: 10 nov. 2020.

4. Lemos KCR, et al. Práticas científicas dos enfermeiros das clínicas de hemodiálise. Revista Enferm. UFPI, Piaui. 2015; 2 (4): 69-75.

5. Mendes KDS, Silveira RCCP, Galvao CM. Revisão integrativa: método de pesquisa para a incorporação de evidências na saúde e na enfermagem. Texto Contexto - Enferm. [online]. 2008; 17 (4): 758-764. Disponivel em: http://dx.doi.org/10.1590/S010407072008000400018. Acesso em: 10 de dez 2020.

6. Ursi ES. Prevenção de lesões de pele no perioperatório: revisão integrativa da literatura. (Dissertação). Ribeirão Preto (SP): Escola de Enfermagem de Ribeirão Preto, 2005.

7. Hames, I. Publicação Acadêmica e Profissional. 15-52, Chandos Publishing, 2012.

8. Hashimoto PC, Ciaccio MCM, Guerra, GM. A tendência do papel do professor no processo de aprendizagem. Revista Nursing (São Paulo). 2018; 21 (24): 2264-2271.

9. Roza RH, et al. Escala de Estilos de Aprendizagem em Situações de Uso de Tecnologias: Estrutura Interna. Revista Avaliação Psicológica. 2018; 17 (2): 223-232.

10. River J et al. A systematic review examining the effectiveness of blending technology with team-based learning. Nurse Education Today, [s.I.]. 2016; 45: 185-192. Elsevier BV.

11. Shin H, Sok S, Hyun KS, Kim MJ. Competency and an active learning program in undergraduate nursing education. Journal of Advanced Nursing. 2015; 71(3), 591-598.

12. Cox SR. Technology to enhance in-class discussions and student participation at a multi-campus program. Currents In Pharmacy Teaching And Learning, Missouri-kansas. 2019; 11 (7): 719-722. Elsevier BV.

13. Gleason SE et al. Five years of lesson modification to implement non-traditional learning sessions in a traditional-delivery curriculum: A retrospective assessment using applied implementation variables. Currents In Pharmacy Teaching And Learning, [s.l.]. 2017; 9 (2): 237245. Elsevier BV.

14. Moraros J et al. Flipping for success: evaluating the effectiveness of a novel teaching approach in a graduate level setting. Bmc Medical Education, [s.I.]. 2015; 15(1): 15-27. Springer Nature.

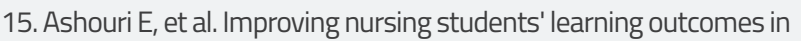
fundamentals of nursing course through combination of traditional and e-learning methods. Iranian Journal Of Nursing And Midwifery Research, [s.l.]. 2018; 23 (3): 217-221. Medknow.

16. Brunner M et al. An eHealth Capabilities Framework for Graduates and Health Professionals: Mixed-Methods Study. Journal Of Medical Internet Research, [s.I.]. 2018; 20 (5): 1-9. JMIR Publications Inc.

17. Carvalho DPSRP, et al. Strategies used for the promotion of critical thinking in nursing undergraduate education: A systematic review. Nurse Education Today, [s.l.]. 2017; 57: 103-107. Elsevier BV.

18. Petit-Dit-Dariel O, Wharrad H, Windle R. Using Bourdieu's theory of practice to understand ICT use amongst nurse educators. Nurse Education Today, [s.l.]. 2014; 34 (11): 1368-1374. Elsevier BV.

19. Wanderley TPSP et al. Docência em saúde: tempo de novas tecnologias da informação e comunicação. Revista Eletrônica de Comunicação, Informação e Inovação em Saúde, [s.l.]. 2018; 12 (4): 488-501. Instituto de Comunicacao e Informacao Cientifica e Tecnologica em Saude.

20. Assis MP, Almeida MEB. Learning Design and Technologies: Creating Collaborative Environments for the Learning Process. Revista Psicologia da Educação (São Paulo). 2017; 44: 47-56.

21. Mcnicholl A, Casey H, Desmond D, Gallagher P. The impact of assistive technology use for students with disabilities in higher education: a systematic review. Disability and Rehabilitation: Assistive Technology. 2019; 1-14. 